\title{
Ethnicity-related differences in inguinal canal dimensions between African and Caucasian populations and their potential impact on the mesh size for open and laparoscopic groin hernia repair in low-resource countries in Africa
}

\author{
Kryspin Mitura ${ }^{1}$, Stawomir Kozieł ${ }^{2}$, Michat Pasierbek ${ }^{3}$ \\ ${ }^{1}$ Department of General Surgery, Hospital, Siedlce, Poland \\ ${ }^{2}$ General Surgery Department, Beskid Centre of Oncology - Municipal Hospital, Bielsko-Biala, Poland \\ ${ }^{3}$ Department of Paediatric Surgery, Hospital No. 1 in Zabrze, Silesian Medical University in Katowice, Poland
}

Videosurgery Miniinv 2018; 13 (1): 74-81

DOI: https://doi.org/10.5114/wiitm.2018.72579

\begin{abstract}
Introduction: Access to surgery in Africa is significantly limited. Treatment outcomes in Africa differ significantly compared to those achieved in Europe or the US. Therefore, to popularise tension-free repair, it is essential to determine the economically justified mesh size for the African population.

Aim: To conduct anthropometric evaluation of the inguinal canal in African and European patients to determine its potential consequences for the mesh size for open and laparoscopic hernia repair.

Material and methods: The measurements were made in 44 adult males in Africa (group I) and were compared to measurements in 45 consecutive Caucasian males (group II). The mean age of patients was respectively 48.3 and 51.2 years.

Results: There was no statistically significant difference in the internal ring diameter between groups $(2.2 \mathrm{vs} .2 .1 \mathrm{~cm}$; $p=0.58)$. The distance between the pubic tubercle and the inferomedial border of the internal inguinal ring was significantly shorter in group I (3.8 vs. $5.1 \mathrm{~cm} ; p<0.001)$. A similar difference was found in the length of transverse arch aponeurosis (2.9 vs. $4.0 \mathrm{~cm} ; p<0.001$ ). The distance between the pubic tubercle and anterior superior iliac spine in group I was approximately $2 \mathrm{~cm}$ shorter on each side (10.0 vs. $11.8 \mathrm{~cm} ; p<0.001)$.

Conclusions: The anatomical differences in inguinal dimensions between Central African and European populations support the potential need to adjust the standard size of synthetic mesh used for hernia repair to the needs of local populations. The significantly smaller dimensions of the inguinal canal in African males may allow the use of smaller meshes.
\end{abstract}

Key words: Africa, inguinal canal, hernia repair, mesh size, low-resource country.

\section{Introduction}

Access to surgery in Africa is significantly limited. This reduced access can be attributed to insufficient human resources, a small number of public hospitals spread across large distances throughout the conti- nent and limited patient transport options [1, 2]. As a result, treatment outcomes in Africa differ significantly from those achieved in Europe or the US. Humanitarian medical missions have been organised to improve the availability of inguinal hernia repair

Kryspin Mitura MD, PhD, Department of General Surgery, Hospital, 15 Starowiejska St, 08-110 Siedlce, Poland, phone: +48 602809035 , 
in Africa and to limit the number of avoidable deaths with timely and appropriate treatment. These cases mainly involve European surgeons, who use their experience to deliver intensive surgical treatment along with local staff training in selected African hospitals [3, 4].

Considering that the prevalence of inguinal hernia in adult Africans is ten-fold higher than that in Europe, large, neglected scrotal hernias are not uncommon in Africa [5]. These hernias impair daily function and limit the work capability of individuals who are typically the only breadwinners in their families. Due to insufficient funding for medical care in Africa, only 25 patients with inguinal hernia per 100,000 inhabitants receive proper surgical treatment, whereas the incidence rate is 60-175 cases per 100,000 inhabitants. As a result, the gap between the number of individuals who need treatment and those actually receiving it increases each year. Tension repair is typically performed since the cost of contemporary synthetic mesh and lack of a distribution network for such devices restricts common use of the Western gold standard procedure, which is tension-free repair. The Lichtenstein procedure that is recommended for open approach inguinal hernia repair usually requires different synthetic mesh implants. Unfortunately, hospitals in low-resource countries lack the financial resources to offer different mesh sizes. Therefore, to popularise tension-free repair it is essential to determine the optimum, universal, economically justified mesh size for that population [6].

\section{Aim}

The aim of this study was to present the intraoperative anthropometric evaluation of inguinal canal structures in African patients undergoing groin hernia repair and to determine the consequences of the anatomical dimensions for the size of hernia mesh implants in open and laparoscopic repairs.

\section{Material and methods}

In January 2016, a humanitarian medical mission was sent to Tanzania. As a part of the mission, three European surgeons performed inguinal hernia repair at the healthcare centre of Kiabakari, Mara region. Medical advice and treatment were delivered to local residents, especially poor villagers, who could not access healthcare services in their country. The sur- gical team brought all necessary devices and instruments with them. All adult patients with suspected or diagnosed inguinal hernias were consulted, and if the diagnosis was confirmed, a surgical repair was scheduled. A total of 89 procedures were performed, and 47 of them were inguinal hernia repairs that were mostly Lichtenstein procedures. All procedures were performed under local anaesthesia. During the procedure, the inguinal canal was accessed, and several dimensions were measured using a sterile ruler to determine the size of the inguinal area, which was referred to as Hessert's triangle [7]. This was the area that was to be secured with the polypropylene mesh to maintain a specific lateral margin (Figure 1). The dimensions were determined after the hernia sac was reduced and the inguinal canal floor with internal inguinal ring was restored, facilitating adequate measurements. The measured dimensions included:

- Internal ring diameter,

- Length of the inguinal ligament between the pubic tubercle and medial margin of the internal ring,

- Length of the transverse arch aponeurosis from the pubic tubercle up to a point at the level of the inferior border of the internal inguinal ring,

- Distance between the midpoint of the inguinal ligament and transverse arch aponeurosis.

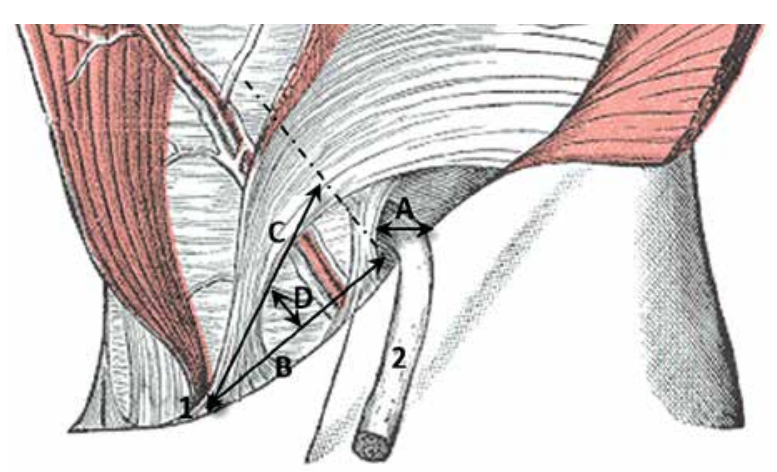

Figure 1. Diagram showing the internal dimensions of the inguinal canal. A - internal ring diameter, $\mathrm{B}$ - length of inguinal ligament between public tubercle and medial margin of internal ring, $\mathrm{C}$ - length of transverse arch aponeurosis from the public tubercle up to a point at the level of the inferior border of the internal inguinal ring, $D$ - distance between midpoint of inguinal ligament and transverse arch aponeurosis, 1 pubic tubercle, 2 - spermatic cord 


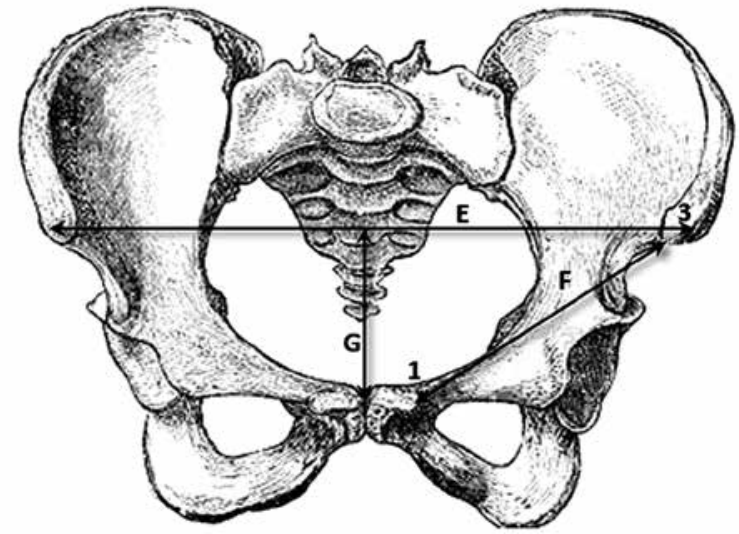

Figure 2. Diagram showing the external dimensions of the pelvis. $\mathrm{E}$ - length of inguinal ligament between pubic tubercle and anterior superior iliac spine, F - distance between anterior superior iliac spines (interspinous line), $\mathrm{G}$ - distance between midpoint of interspinous line and public symphysis, 1 - pubic tubercle, 3 - anterior superior iliac spine

Postoperatively, specific pelvic dimensions were measured using metal callipers (Figure 2), including:

- Distance between the pubic tubercle and anterior superior iliac spine (inguinal ligament),

- Distance between the two anterior superior iliac spines (interspinous line),

- The vertical segment between the interspinous line and pubic tubercle (pubic arch).

The measurements were made in a sample of 44 adult African males (group I) and were compared to measurements taken in 45 consecutive cases of adult Caucasian males treated for inguinal hernia in Europe (group II). All measurements were taken by the same surgical team.

Group I included patients with mean age of 48.3 \pm 20.4 years (18-82). Only 2 patients had direct hernia. All others had indirect hernias. Eighteen patients had extensive scrotal hernias (lower pole of the hernia sac extending at least $10 \mathrm{~cm}$ below the pubic tubercle). The mean age of patients in group II was $51.2 \pm 17.9$ years (18-79); 33 of them had indirect hernias and 12 had direct hernias, including 7 cases of scrotal hernia. There were no significant differences regarding age. The differences in type of hernia (direct or indirect) are considered to have no influence on the anthropometric measurements since these parameters are mainly based on bony structures and embryologic development of musculofascial planes.

\section{Statistical analysis}

Descriptive statistics included mean, standard deviation (SD), and range for continuous variables and number and percentages for categorical variables. Exploratory data analysis was used to assess the normal distribution of continuous variables. Student's $t$-test was used to assess differences in anthropometric measurements between African and European populations. A $p$-value less than 0.05 was considered statistically significant.

\section{Results}

Table I shows the results of metric measurements in both populations. There were no statistically significant differences in internal ring diameter between groups I and II $(2.2 \mathrm{~cm}$ vs. $2.1 \mathrm{~cm}$; $p=0.58)$. The distance between the pubic tubercle and the inferomedial border of the internal inguinal ring was significantly shorter in group I $(3.8 \mathrm{~cm}$ vs. $5.1 \mathrm{~cm} ; p<0.001)$. Similar differences between groups I and II were observed in the length of the transverse arch aponeurosis extending from the pubic tubercle up to a point at the level of the inferior border of the internal inguinal ring $(2.9 \mathrm{~cm}$ vs. $4.0 \mathrm{~cm} ; p<0.001)$.

The accuracy of internal measurements was verified with external pelvic dimensions. The mean length of the interspinous line in African subjects (group I) was shorter compared to the same distance measured in Caucasian subjects from Europe (group II) by $3.6 \mathrm{~cm}(22.2 \mathrm{~cm}$ vs. $25.8 \mathrm{~cm}$; $p<0.001)$. The distance between the pubic tubercle and anterior superior iliac spine (inguinal ligament length) in group I was approximately $2 \mathrm{~cm}$ shorter on each side compared to group II $(10.0 \mathrm{~cm}$ vs. $11.8 \mathrm{~cm}$; $p<$ $0.001)$. The distribution of both variables is shown in Figure 3.

\section{Discussion}

Poor access to specialist surgical care in Africa is reflected by data demonstrating unsatisfactory treatment outcomes in patients with inguinal hernias [8]. A low number of elective procedures results in $65 \%$ of inguinal hernia repairs being performed as emergency procedures due to strangulation of hernia [9]. Strangulated hernias constitute only $1-5 \%$ of all hernias repaired in Europe or the US [10]. In Africa, strangulated hernias are usually treated late 
Table I. Measurements of distances between landmarks of the inguinal canal and pelvis

\begin{tabular}{|c|c|c|c|c|c|c|c|c|c|}
\hline \multirow[t]{2}{*}{ Variable } & \multicolumn{4}{|c|}{ Group I } & \multicolumn{4}{|c|}{ Group II } & \multirow[t]{2}{*}{$P$-value } \\
\hline & $\begin{array}{c}\text { Mean } \\
{[\mathrm{cm}]}\end{array}$ & SD & $\begin{array}{l}\text { Min } \\
{[\mathrm{cm}]}\end{array}$ & $\begin{array}{l}\text { Max } \\
{[\mathrm{cm}]}\end{array}$ & $\begin{array}{c}\text { Mean } \\
{[\mathrm{cm}]}\end{array}$ & SD & $\begin{array}{l}\text { Min } \\
{[\mathrm{cm}]}\end{array}$ & $\begin{array}{l}\text { Max } \\
{[\mathrm{cm}]}\end{array}$ & \\
\hline \multicolumn{10}{|l|}{ Internal: } \\
\hline Internal ring diameter & 2.2 & 1.08 & 1.0 & 4.5 & 2.1 & 0.41 & 1.0 & 3.0 & 0.58 \\
\hline $\begin{array}{l}\text { Length of the inguinal } \\
\text { ligament between the } \\
\text { pubic tubercle and the } \\
\text { medial margin of the } \\
\text { internal ring }\end{array}$ & 3.8 & 0.68 & 2.5 & 5.5 & 5.1 & 0.57 & 4.0 & 6.5 & $<0.001$ \\
\hline $\begin{array}{l}\text { Length of the trans- } \\
\text { verse arch aponeurosis } \\
\text { from the pubic tubercle } \\
\text { up to a point at the } \\
\text { level of the inferior } \\
\text { border of the internal } \\
\text { inguinal ring }\end{array}$ & 5.2 & 1.01 & 2.5 & 7.0 & 5.6 & 0.76 & 4.0 & 7.0 & 0.11 \\
\hline $\begin{array}{l}\text { Distance between } \\
\text { the midpoint of the } \\
\text { inguinal ligament } \\
\text { and transverse arch } \\
\text { aponeurosis }\end{array}$ & 2.9 & 0.67 & 2.0 & 5.0 & 4.0 & 0.66 & 3.0 & 6.0 & $<0.001$ \\
\hline \multicolumn{10}{|l|}{ External: } \\
\hline $\begin{array}{l}\text { Length of the inguinal } \\
\text { ligament between the } \\
\text { pubic tubercle and the } \\
\text { anterior superior iliac } \\
\text { spine }\end{array}$ & 10.0 & 1.29 & 8.0 & 13.0 & 11.9 & 1.19 & 9.5 & 14.5 & $<0.001$ \\
\hline $\begin{array}{l}\text { Distance between the } \\
\text { anterior superior iliac } \\
\text { spines (interspinous } \\
\text { line) }\end{array}$ & 22.2 & 2.31 & 19 & 28 & 25.8 & 1.48 & 22 & 28 & $<0.001$ \\
\hline $\begin{array}{l}\text { Distance between the } \\
\text { midpoint of the inter- } \\
\text { spinous line and the } \\
\text { pubic symphysis }\end{array}$ & 6.8 & 1.56 & 4 & 10 & 8.2 & 1.27 & 6 & 11 & $<0.001$ \\
\hline
\end{tabular}

and are typically treated $72 \mathrm{~h}$ after strangulation. As a result, segmental bowel resection is needed in approximately $24 \%$ of cases and the postoperative mortality rate in these patients is $6 \%$. Among patients who do not reach hospital in time with strangulated hernias, the mortality rate is 87 deaths per 100 cases $[3,4]$.

Such dramatic epidemiological data have led Western surgeons to participate in humanitarian medical missions to Africa. In recent years, we have had the opportunity to participate in several such missions to different African countries, including Tanzania, Ghana, Ethiopia, Gambia, and the Ivory
Coast [11]. When performing inguinal hernia repair in patients from those countries, we noticed that the mesh implants routinely used in Europe (sized $10 \times 15 \mathrm{~cm}$ ) had to be trimmed. Otherwise, it was impossible to place them flat due to their excessive size. However, we were unable to find publications addressing the anthropometric dimensions of the inguinal area in both the Caucasian populations of Europe and the black populations of Central Africa. Our results support the hypothesis that the observed differences in suitable mesh sizes may be due to differences in dimensions of the anatomical structures between the two populations. 


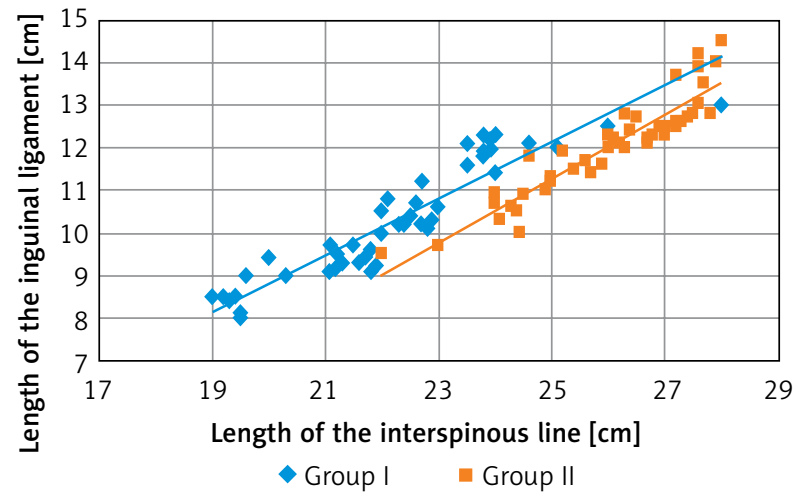

Figure 3. Diagram showing the relationship between the length of the inguinal ligament and the length of the interspinous line

The majority of published studies have compared pelvic dimensions in childbearing women, so measurements of smaller pelvises were typically taken. Based on metric analyses of magnetic resonance imaging (MRI) scans in primigravidae, Handa et al. found a significantly smaller inlet and outlet diameter in African-American women compared to Caucasian women $(10.0 \mathrm{~cm}$ vs. $10.7 ; p<0.001$ and $11.8 \mathrm{~cm}$ vs. $12.3 \mathrm{~cm} ; p<0.001$ ) [12]. Similar results were published by Baragi, who reported that the pelvic floor area in African-American women was much smaller than that in Caucasian women - by 5.1\% [13]. Although these results confirmed the presence of ethnicity-related differences in pelvis size, they could not be extrapolated to males, who constitute the majority of patients with inguinal hernias. Forensic anthropologists are another group of professionals who have studied the differences in pelvic sizes between sexes and ethnicities. However, the majority of forensic anthropology research was carried out around the 1950s before the onset of synthetic mesh repair. Therefore, the parameters essential for herniology were not actually studied, although the significantly larger pelvic size in the Caucasian race compared to the African race was confirmed [14, 15]. Additionally, the majority of available research was difficult to reproduce due to it being based on hard-to-locate landmarks, such as assessing the dimension of the wing of the ilium based on a single-point landmark situated within the acetabulum. Based on objective measurements of 400 complete pelvic bones of South African residents, Patriquin et al. obtained reliable and credible data on the actual differences in pelvic size between Caucasian and African males and fe- males [16]. The authors found that all nine assessed pelvic parameters were significantly smaller in black subjects compared to Caucasian subjects $(p<0.001)$. Furthermore, the pelvic measurements offered high positive predictive value (97\%) for identifying the bones of black males. These results were consistent with our findings. The computed distance between the pubic tubercle and anterior superior iliac spine was significantly shorter in African males compared to Caucasian males (i.e., there was a mean difference of $19 \mathrm{~mm} ; p<0.001)$, whereas the difference in the interspinous line length was $36 \mathrm{~mm}(22.2 \mathrm{~cm}$ vs. $25.8 \mathrm{~cm}$ in African and Caucasian males, respectively; $p<0.001$ ). The interspinous line length in Caucasian males was similar to the length measured by Steyn, who - in her study of Greek males - reported a distance between two anterior superior iliac spines of $27.7 \pm 1.4 \mathrm{~cm} \mathrm{[17].}$

The external pelvic measurements indirectly influence the relative distance between the individual structures of the inguinal canal. Thus, they affect the size of mesh implants that may be used during open and laparoscopic hernia repair in those patients. In our sample, the inguinal canal of African patients was approximately $13 \mathrm{~mm}$ shorter than that of Caucasian patients $(3.8 \mathrm{~cm}$ vs. $5.1 \mathrm{~cm}$, respectively; $p<0.001)$. Peri et al. reported a similar length of the inguinal canal in Caucasian males $(4.7 \mathrm{~mm})$ [18]. Furthermore, Peri et al. reported that the internal inguinal ring diameter ranged from $1.5 \mathrm{~cm}$ to $2.5 \mathrm{~cm}$, which was consistent with our findings $(2.2 \mathrm{~cm}$ in group I and $2.1 \mathrm{~cm}$ in group II). Considering the total length of the inguinal ligament in both study groups, we concluded that the distance between the inferior border of the internal inguinal ring and the superior anterior iliac spine was shorter in African males than in Caucasian males $(4.0 \mathrm{~cm}$ vs. $4.7 \mathrm{~cm})$. The direct clinical implication of these differences in open inguinal hernia repair is that it is often impossible to secure the lateral tails of the mesh if the mesh extends more than 3-4 cm outside the internal ring in African patients. However, this distance was shorter than distances commonly used in clinical practice in Europe or the US. Thus, considering that inguinal hernia repair in an African patient requires an overlap of $2 \mathrm{~cm}$ above the pubic tubercle, securing the entire length of the inguinal canal (at a mean length of $3.8 \mathrm{~cm}$ ) and the internal inguinal ring (the mean diameter of $2.2 \mathrm{~cm}$ ) with mesh tails extending approximately $3 \mathrm{~cm}$ outside the internal ring, the entire mesh implant should 
not be longer than $11 \mathrm{~cm}$, which correlates with the measurements we took in Tanzanian patients.

A low pubic arch, which is the vertical distance between the interspinous line and pubic symphysis, is an established factor that affects the incidence of hernias [19]. In our sample, we found a significantly shorter distance between the pubic tubercle and interspinous line in African males compared to Caucasian males $(6.8 \mathrm{~cm}$ vs. $8.2 \mathrm{~cm} ; p<0.001)$. Along with malnutrition, collagen deficiency and hard manual labour are other anatomical factors that contribute to the higher incidence of hernias in the African population [20]. Our measurements in the Caucasian population were in line with a study by Lopez-Cano et al., who examined 17 male corpses and found the mean distance between the pubic tubercle and the interspinous line to be $8.17 \pm 1.42 \mathrm{~cm}$ [21]. They observed that the length of the inguinal ligament and pubic triangle increased with the lower pubic arch. It should be noted, though, that this observation was only true in the Caucasian population. It did not apply to African patients, who had narrower pelvises, and the threshold value to describe the pubic arch as "low" $(7.5 \mathrm{~cm})$ should be redefined in a black population and established at a level of $6 \mathrm{~cm}$.

The measurements of the inguinal canal demonstrated a similar tendency for the African population, and there was a shorter vertical pelvic dimension in the suprapubic area. Whereas the length of the transverse arch aponeurosis was similar in groups I and II (5.2 cm vs. $5.6 \mathrm{~cm} ; p=0.11)$, the transverse dimension of the inguinal canal measured at halflength was 2.9 in African subjects and 4.0 in Caucasian subjects $(p<0.001)$. Rabe et al. found that the mean width of the inguinal canal in patients from the Philippines was $4.0 \mathrm{~cm}$, which was similar to our Caucasian population [22]. This was the area of Hessert's triangle that needed to be secured with mesh with appropriate overlap [7]. Considering the narrow inguinal canal and lower pubic arch, we can suggest that instead of the European standard width size of 7.0-7.5 cm, the mesh implants in open repair intended for African patients should be approximately $1.5 \mathrm{~cm}$ shorter, so the $6.0 \mathrm{~cm}$ mesh width might be appropriate. An obvious limitation of this study is the small number of patients included, therefore definitely the validity of the results is limited until further studies confirm our observations.

Although the use of laparoscopic methods in groin hernia repair is practically still almost unavail- able in most African countries, it appears that surgeons should be aware of the possibility of similar dimensional differences as in open repairs when implementing miniinvasive techniques in the coming years. The shorter length of Cooper's ligament and low pubic arch can potentially create difficulty in placing a large $10 \times 15 \mathrm{~cm}$ mesh in the preperitoneal space. At this point, trimming the mesh may be the only way to fit the material in the preperitoneal area. Nevertheless, the need to use mesh fixation may still remain the surgeon's decision as with other laparoscopic hernia operations, without compromising the effectiveness of the method [23]. Of course, these considerations are merely a baseline for further studies, as laparoscopic approaches for patients in low resource countries are introduced in selected leading centres only. At present, the main goal of providing surgical medical care in Africa is to prevent the serious complications of hernias left untreated. As the number of treatment centres and their experience increase, more attention should be paid to the quality of treatment (chronic pain, recurrences), and at some point then there may be a need for laparoscopic procedures [24].

Numerous humanitarian medical missions to Central Africa organised by surgeons from Western European countries and the US have placed increasing emphasis on delivering training in tension-free inguinal hernia repair techniques to local surgeons, along with performing surgery and providing local hospitals with appropriate equipment and medical instruments. The high costs of synthetic hernia mesh implants still preclude the use of contemporary surgical methods by local surgeons in Africa. To reduce the cost, attempts have been made to replace commercially available mesh implants with sterilised mosquito nets. However, these nets have not been studied extensively enough to be recommended for common use [25]. As a result, hospitals and other health centres in Africa still need mesh implants with appropriate sizes. African hospitals do not have different types and sizes of hernia mesh implants available, which is often the case with hospitals in Western countries. Therefore, it is necessary to determine a universal size for the mesh so the cost can be economically justified. Routine use of mesh that is too large, which needs to be trimmed during the surgery, results in wasting part of the device and the money spent on it, which could have been allocated elsewhere. For that reason, establishing the 
optimum mesh size sufficient to protect the patient from hernia recurrence may improve the availability of mesh implants in developing countries. Due to the limited number of patients in our sample, similar analyses need to be conducted during other humanitarian medical missions to other countries.

The standard mesh size of $7.0 \times 15.0 \mathrm{~cm}$, which is recommended by the Lichtenstein Hernia Institute, can be trimmed by $2.0 \mathrm{~cm}$ and is perfectly suitable for Caucasian males [26, 27]. However, due to the differences in pelvic structure and significantly smaller size of individual structures as well as the inguinal canal, this mesh size is too large to be used routinely in African males. Objective measurements of inguinal canal structures confirmed that a mesh size of $6.0 \times 11.0 \mathrm{~cm}$ was usually suitable for most inguinal hernia repairs in patients from Central Africa, while the principles of 1.5 to $2 \mathrm{~cm}$ of tubercle coverage, 2-3 cm coverage of the medial conjoined tendon, and $4-5 \mathrm{~cm}$ of cephalad overlap was achieved. Providing mesh implants of this size could decrease the unit price of the device and improve access to surgical hernia repair according to world standards.

The limitation of the study is that it is a single-centre analysis based on a small group of patients. The mesh sizes to be used in the African population are purely speculative as the study did not address the issue of clinical outcomes in its methodology. Therefore, it is necessary to point out that the results of the study only suggest that mesh sizes for the African population may be reduced in dimensions, but this issue must be explored and justified by further studies.

\section{Conclusions}

Poor access to inguinal hernia repair in developing countries implies the need to search for economically justified solutions for popularising the Lichtenstein procedure. The anatomical differences in inguinal canal dimensions between Central African and European populations indicate that it is necessary to adjust the standard size of synthetic mesh used for inguinal hernia repair to the needs of local populations. The significantly smaller dimensions of the inguinal canal in African males make it possible to use smaller mesh implants.

\section{Conflict of interest}

The authors declare no conflict of interest.

\section{References}

1. Dare L, Buch E. The future of health care in Africa. Br Med J 2005; 331: 1-2.

2. Lucas AO. Human resources for health in Africa. Br Med J 2005; 331: 1037-8.

3. Sanders DL, Kingsnorth AN. Operation hernia: humanitarian hernia repairs in Ghana. Hernia 2007; 11: 389-91.

4. Kingsnorth AN, Oppong C, Akoh J, et al. Operation hernia to Ghana. Hernia 2006; 10: 376-9.

5. Nordberg EM. Incidence and estimated need of caesarean section, inguinal hernia repair and operation for strangulated hernia in rural Africa. Br Med J 1984; 289: 92-3.

6. Ferraz de Carvalho CA, Souza RR, Fernandes PMP. The relationship between anthropometric parameters and measurements of the human inguinal region. Surg Radiol Anat 1987; 9: 281-5.

7. Abdalla RZ, Mittelstaedt WE. The importance of the size of Hessert's triangle in the etiology of inguinal hernia. Hernia 2001; 5: 119-23.

8. Sanders DL, Porter CS, Mitchell KCD, et al. A prospective cohort study comparing the African and European hernia. Hernia 2008; 12: 527-9.

9. Ohene-Yeboah M. Strangulated external hernias in Kumansi. West Afr Med J 2003; 22: 310-3.

10. Primatesta P, Goldacre MJ. Inguinal hernia repair: incidence of elective and emergency surgery, readmission and mortality. Int J Epidemiol 1996; 25: 502-9.

11. Mitura K, Kozieł S, Pasierbek M. Groin hernia surgery in northern Ghana - humanitarian mission of Polish surgeons in Tamale. Pol Przegl Chir 2015; 87: 16-21.

12. Handa VL, Lockhart ME, Fielding JR, et al. Racial differences in pelvic anatomy by magnetic resonance imaging. Obstet Gynecol 2008; 111: 914-20.

13. Baragi RV, Delancey JO, Caspari R, et al. Differences in pelvic floor area between African American and European American women. Obstet Gynecol 2002; 187: 111-5.

14. Letterman GS. The greater sciatic notch in American Whites and Negroes. Am J Phys Anthropol 1941; 28: 99-116.

15. DiBennardo R, Taylor JV. Multiple discriminant function analysis of sex and race in the postcranial skeleton. Am J Phys Anthropol 1983; 61: 305-14.

16. Patriquin ML, Steyn M. Metric analysis of sex differences in South African black and white pelves. Forensic Sci Int 2005; 147: 119-27.

17. Steyn M, Iscan MY. Metric sex determination from the pelvis in modern Greeks. Forensic Sci Int 2008; 179: 86.e1-6.

18. Peri G, Farina F, Marciano V, et al. Clinical and anatomic features of the inguinal canal during hernia. Ital J Anat Embryol 1996; 101: 69-80.

19. Ledinsky M, Matejcic A, De Syo D, et al. Some structural characteristics of the inguinal region in the Northern Croatia. Coll Antropol 1998; 22: 515-24

20. Zinanovic S. The anatomical basis for the high frequency of the inguinal and femoral hernia in Uganda. East Afr Med J 1968; 45: 41-6.

21. Lopez-Cano M, Munhequete EG, Hermosilla-Perez E. Anthropometric characteristics of the pubic arch and proper function 
of the defense mechanisms against hernia formation. Hernia 2005; 9: 56-61.

22. Rabe R, Yacapin CPR, Buckley BS, et al. Repeated in vivo inguinal measurements to estimate a single optimal mesh size for inguinal herniorrhaphy. BMC Surgery 2012; 12: 19.

23. Pawlak M, Bury K, Smietanski M. The management of abdominal wall hernias - in search of consensus. Videosurgery Miniinv 2015; 10: 49-56.

24. Klobusicky P, Hoskovec D. Reduction of chronic post-herniotomy pain and recurrence rate. Use of the anatomical self-gripping ProGrip laparoscopic mesh in TAPP hernia repair. Preliminary results of a prospective study. Videosurgery Miniinv 2015; 10: 373-81.

25. Koziel S, Mitura K, Papaj P, et al. Tension-free mosquito net mesh inguinal hernioplasty - an alternative exclusively for the Third World? Pol Przegl Chir 2015; 87: $102-7$.

26. Mitura K, Romanczuk M. Redundant modifications of Lichtenstein technique in hernia repair - a descriptive study of practising surgeons in Poland. Videosurgery Miniinv 2009; 4: 1-5.

27. Amid PK. The Lichtenstein in 2002: an overview of causes of recurrence after Lichtenstein tension-free hernioplasty. Hernia 2003; 7: 13-6.

Received: 16.08.2017, accepted: 3.10.2017 